\title{
Cinta de náilon como cerclagem óssea - estudo experimental em coelhos e ratos
}

\section{Nylon bands as bone cerclage - experimental study in rabbits and rats}

\author{
Claudia Valéria Seullner Brandão'; Pedro Afonso Urgel Martins Costa Antunes²; \\ Caroline Abreu Estanislau²; Maria Jaqueline Mamprim²; Leandro Teixeira²; \\ Carlos Roberto Padovani ${ }^{3}$; Paulo Vinicius Tertuliano Marinho ${ }^{4 *}$; \\ Bruno Watanabe Minto ${ }^{5}$
}

\section{Resumo}

\begin{abstract}
O presente trabalho teve como objetivo avaliar a biocompatibilidade da aplicação da cinta de náilon comercial como cerclagem óssea e compará-la com a cerclagem tradicional de fio aço. Foram utilizados 21 coelhos e 15 ratos, sadios e sem alterações no aparelho locomotor. Nos coelhos, uma cinta de náilon comercial foi aplicada no membro pélvico direito (SN) e um fio de aço cirúrgico $\mathrm{n}^{\circ} 0$ no esquerdo (SA). Nos ratos, o SN foi o membro esquerdo e o SA o direito. Ambas as espécies foram separadas em três grupos de acordo com o período final de avaliação. Os animais foram avaliados por exames clínico, radiográfico, macroscópico, e histológico aos 30,60 e 150 dias pós-implantação. Todos os animais apresentaram função locomotora normal; na avaliação clínica e macroscópica não foram observadas diferenças entre os grupos. Em ambas as espécies, as alterações radiográficas peri-implante foram levemente superiores no implante náilon em relação ao fio de aço. Não foram observadas diferenças na análise microscópica entre os subgrupos náilon e aço, em ambas as espécies. Pode-se concluir que a cinta de náilon não causa reações de corpo estranho nem alterações histológicas no osso normal em ratos e coelhos.
\end{abstract}

Palavras-chave: Ortopedia, fraturas, fêmur, Oryctolagus cuniculus, Rattus norvegicus albinus

\begin{abstract}
The aim of this study was to evaluate the biocampatibility of the commercial nylon band used as bone cerclage, comparing with traditional metal cerclage wires. Twenty one rabbits and fifteen rats, healthy and without orthopedics problems were used. A commercial nylon band and a surgical stainless steel wire "0" were used in right (SN) and left (SA) rabbits's hindlimb, respectively. Otherwise, on rats $\mathrm{SN}$ was on the left hindlimb, and SA on the right hindlimb. Both experimental species were divided into three groups according to the end of the evaluation period. Clinical, radiographic, macroscopic and histologically evaluation were performed at 30,60 and 150 days postoperatively. All the animals showed normal limb function differences between groups were not observed at clinical and macroscopic

\footnotetext{
${ }^{1}$ Prof. da Faculdade de Medicina Veterinária da FMVZ/UNESP, Botucatu, SP. E-mail: valeriasb@fmvz.unesp.br

${ }^{2}$ Pesquisadores da FMVZ/UNESP, Botucatu, SP. E-mail: peantunesvet@yahoo.com.br; caestanis@yahoo.com.br; jaquelinem@ fmvz.unesp.br; leteixeira-vet@hotmail.com

${ }^{3}$ Médico Veterinário do Instituto de Biociências, IB, UNESP, Campus de Botucatu, Botucatu, SP. E-mail: padovanicr@ibb.ubesp.br

${ }^{4}$ Discente de Mestrado, Pesquisador da Universidade Estadual de Londrina, UEL, Londrina, PR. E-mail: paulo.veter@gmail.com

${ }^{5}$ Prof. da Faculdade de Ciências Agrárias e Veterinária, FCAV/UNESP, Jaboticabal, SP. E-mail: brunobtu@yahoo.com.br

* Autor para correspondência
} 
evaluation. On both species, radiographic changes were slightly higher on SN. Histological differences were not observed in both species. Nylon bands do not cause foreign body reaction and other histological changes in rabbits and rats.

Key words: Orthopedics, fracture, femur, Oryctolagus cuniculus, Rattus norvegicus albinus

\section{Introdução}

A aproximação de fragmentos ósseos afastados é considerada uma das principais manobras realizadas na redução de fraturas, exigindo-se no procedimento habilidade do cirurgião. Recomendam-se, desta forma, técnicas e material cirúrgico que sejam eficazes e práticos, proporcionando maior agilidade no período trans-operatório e auxiliando na consolidação óssea (RAISER et al., 1981; MIRANDA et al., 2006).

A cinta de náilon tem sido um método alternativo para reconstituição de fraturas de ossos longos, associada com pinos intramedulares ou placas (SCHMIDT; DAVIS, 1981; KIRBY; WILSON, 1991; CARRILLO et al., 2005). O dispositivo é uma cinta de náilon 6.6 (poliamida) comercial, material biologicamente inerte, empregada rotineiramente na engenharia elétrica e adaptada para uso em vários procedimentos cirúrgicos (SORBELLO; GIUDUGLI; ANDRETTO, 1999; MIRANDA et al., 2004; LIMA et al., 2010).

Embora Rhinelander e Stewart (1983) tenham afirmado que o mecanismo de trava da cinta de náilon é muito maior que o fecho do fio de aço e que, portanto, requer mais espaço nos tecidos, para Schmidt e Davis (1981), a cinta de náilon, em comparação com o fio de aço, apresenta como vantagens a facilidade na sua aplicação e menor lesão causada aos tecidos ao seu redor. O dispositivo também tem sido utilizado com sucesso em cirurgias na cavidade abdominal, na hemostasia preventiva de ovariohisterectomia em cadelas (SILVA et al., 2004; LIMA et al., 2010), em éguas (FRANÇA, 2005) e ainda como método hemostático em cirurgias videoendoscópicas (SORBELLO; GIUDUGLI; ANDRETTO, 1999). Albuquerque (1990) apontou sua excepcional tenacidade, alta resistência ao desgaste, baixo coeficiente de atrito e resistência química excelente. $\mathrm{O}$ autor considera ainda o baixo custo, resistência à alta pressão, amortecimento de vibrações e pesos, elevada resistência ao corte e resistência a agentes químicos como vantagens importantes.

Frente às vantagens acima descritas, a realização de um trabalho experimental utilizando as cintas de náilon, foi estimulada. Este teve como objetivo avaliar, por meio de exame clínico, radiográfico, macroscópico e histopatológico, a biocompatibilidade e o comportamento ósseo frente à aplicação de cerclagem com cinta de náilon e fio de aço, bem como comparar o efeito dos mesmos em biometrias femorais distintas, em animais da espécie Oryctolagus cuniculus (coelhos) e Rattus norvegicus albinus (ratos).

\section{Material e Métodos}

Animais e ambiente de experimentação

Foram utilizados 21 leporídeos da espécie Oryctolagus cuniculus, raça Norfolk, machos, 4 meses de idade e pesando $3 \mathrm{~kg}$; e 15 ratos da espécie Rattus norvegicus albinus, machos, 45 dias de idade e peso 180 gramas, sem alterações no aparelho locomotor. Os coelhos foram mantidos em gaiolas, 60x50x60 cm, com dois animais em cada e os ratos, em caixas de polietileno, 20x30x14 cm, com dois animais em cada; todos os animais foram alimentados com ração comercial própria para a espécie e água ad libitum.

\section{Delineamento experimental}

Para cada espécie foram constituídos três grupos experimentais de sete coelhos e cinco 
ratos, designados de acordo com o período final de avaliação como M30, M60 e M150, o que representou 30, 60 e 150 dias de pós-operatório respectivamente. Sob condições assépticas, os animais foram submetidos à aplicação cirúrgica dos implantes no terço médio dos fêmures. Nos coelhos, uma cinta (abraçadeira) de náilon ${ }^{6}$ (140 X 2,5 mm constituída unicamente de poliamida segundo laudo do fabricante), foi aplicada no membro pélvico direito e um fio de aço cirúrgico ${ }^{7} \mathrm{n}^{\circ} 0$ no esquerdo. Ambos os materiais utilizados foram esterilizados em autoclave. Nos ratos, entretanto, aplicou-se o náilon no membro esquerdo e o aço no direito. Os membros foram designados como subgrupos de acordo com o tipo de material utilizado: subgrupo náilon (SN) e subgrupo aço (SA).

\section{Parâmetros avaliados}

Os animais foram avaliados clinicamente quanto à locomoção, inflamação da ferida e fístulas nos períodos de 24 horas, 30 dias, 60 e 150 dias após a cirurgia. Para os dois últimos parâmetros foi utilizado o escore "0" para indicar ausência e " 1 " presença das alterações. Para a locomoção utilizouse " 0 " para indicar ausência de claudicação, "1" claudicação discreta, "2" claudicação moderada e "3” claudicação intensa (Tabela 1).

Tabela 1. Classificação da deambulação de coelhos e ratos segundo o grau e critério utilizado.

\begin{tabular}{cl}
\hline Grau & \multicolumn{1}{c}{ Critério } \\
\hline 0 & $\begin{array}{l}\text { Claudicação ausente; apoio completo do membro com o animal em estação ou durante atividade física } \\
1\end{array}$ \\
$\begin{array}{l}\text { Claudicação esporádica ao caminhar ou correr, com alívio do peso sobre o membro operado, inclusive } \\
\text { em estação }\end{array}$ \\
$\begin{array}{l}\text { Claudicação constante ao caminhar e não apoio do membro ao correr; apoio incompleto em posição } \\
\text { ortostática }\end{array}$ \\
Apoio incompleto ou ausente durante a locomoção ou em estação
\end{tabular}

Fonte: Modificado de Muzzi et al. (2003).

Realizou-se exame radiográfico dos fêmures antes do procedimento cirúrgico e nos diferentes momentos de avaliação, de acordo com o seu grupo experimental. As projeções craniocaudal $(\mathrm{Cr} C d)$ e mediolateral $(M L)$ foram realizadas com a incidência dos raios em ângulo de $90^{\circ}$ em relação ao maior eixo do fêmur, com o membro em extensão. A osteólise, o volume e a opacidade dos tecidos moles adjacentes ao implante foram avaliados utilizandose escores 0 (ausente) e 1 (presente). Para a reação periosteal, foi utilizado um escore de 0 a 3, no qual " 0 " representa ausência, "1" presença leve (reação periosteal apenas nos bordos do implante), "2"

\footnotetext{
${ }^{6}$ FoxLux - Senes \& Rossi LTDA - Curitiba - PR - Brasil.

${ }^{7}$ Monicron - Cirumédica - SP - Brasil.

${ }^{8}$ Tipental Sódico - Cristália - Itapira - SP.

${ }^{9}$ Isoforine - Cristália - Itapira - SP.
}

moderada (reação periosteal nas bordas do implante com radiopacidade evoluindo concentricamente) e " 3 " presença intensa (reação periosteal nos bordos do implante com radiopacidade estabelecida concentricamente).

De acordo com o momento final de avaliação de cada grupo, foi realizada a eutanásia dos animais. Nos coelhos foi utilizado tiopental ${ }^{8}$ na concentração de $2,5 \%$, na dose de $25 \mathrm{mg} / \mathrm{Kg}$ intravenoso (IV) e após a indução, aplicado cloreto de potássio (KCl) a $19,1 \%$ na dose de $1 \mathrm{mg} / \mathrm{Kg}$, IV. Para os ratos foi utilizado um ambiente hipersaturado por isofluorano?. 
Avaliação macroscópica dos tecidos moles e tecido ósso peri-implante nos fêmures de cada animal foi realizada após incisão dos planos teciduais. Foram avaliadas a proliferação de tecido fibroso, aderência e neoformação óssea. Para isso, foram utilizados os escores de " 0 " a " 3 " ( 0 - ausente, 1 - leve, 2 - moderado e 3 - intenso).

A biometria femoral foi realizada com auxilio a excisão dos fêmures e realizada a sua medição por meio de um paquímetro. Obtiveram-se as medidas das distâncias, em milímetros ( $\mathrm{mm})$, do trocânter menor até a epífise distal, da face cranial à caudal e da medial à lateral do fêmur. Para a realização das últimas duas medidas, foi determinada a distância de $1 \mathrm{~cm}$ acima do local do implante nos coelhos e $0,5 \mathrm{~cm}$ nos ratos.

Para a avaliação histopatológica foi coletado um fragmento ósseo da diáfise de cada fêmur, envolvendo $1 \mathrm{~cm}$ proximal e $1 \mathrm{~cm}$ distal ao implante. Após descalcificação e processamento histológico, as lâminas foram coradas com Hematoxilina Eosina (HE) e analisadas ao microscópio óptico, avaliandose a presença de células inflamatórias, fibrose periosteal, porosidade, proliferação de periósteo, isquemia, atividade osteoblástica e maturidade do tecido, sob o implante. Para estes parâmetros foi atribuído um escore de " 0 " a " 3 " ( 0 - ausente, 1 leve, 2 - moderado e 3 -intenso).

Em todos os parâmetros, os avaliadores não tiveram conhecimento a respeito do grupo na amostra que estava sendo avaliada.

$\mathrm{Na}$ avaliação estatística, para a comparação e avaliação das medidas dos fêmures, variáveis intervalares, foi utilizada a técnica da análise de variância paramétrica para o modelo de medidas repetidas com dois fatores (momento de eutanásia e tipo de material), complementada pelo teste de comparações múltiplas de médias (ZAR, 1999). Para comparação e avaliação das variáveis ordinais, ou seja, aquelas para as quais foi criado um escore de 0 a 3 , foi utilizada a técnica de análise de variância não-paramétrica para o modelo de medidas repetidas com dois fatores (momento de eutanásia e tipo de material), complementado pelo teste de comparações múltiplas de Dunn (ZAR, 1999). Os testes foram aplicados ao nível de 5\% de significância $(\mathrm{p}<0,05)$.

\section{Resultados e Discussão}

\section{Avaliação clínica}

A abordagem cirúrgica lateral ao fêmur promoveu amplo acesso à diáfise femoral, bem como colocação adequada dos implantes. Semelhante ao descrito por Schmidt e Davis (1981) e Miranda et al. (2006), observou-se que o implante de náilon é de aplicação rápida e fácil quando comparada à aplicação da cerclagem por fio de aço, minimizando assim as lesões aos tecidos moles.

No experimento realizado por Carrillo et al. (2005), no qual foram utilizadas cintas de náilon associadas a pinos intramedulares para reparo de fraturas oblíquas em coelhos, os animais apresentaram movimento satisfatório dos membros após 24 horas da cirurgia. Do mesmo modo, embora sem indução de fratura, neste experimento, desde o pós-cirúrgico até o momento final de cada grupo experimental, não foram observadas alterações na locomoção, ou seja, todos os animais apresentaram escore " 0 ", mostrando a adequação na aplicação dos implantes e ausência de interferência clínica dos mesmos nos membros operados. No oitavo dia do pós-operatório, foi observada a cicatrização da ferida cirúrgica em todos os animais sem edema, secreção e deiscência dos pontos.

\section{Avaliação radiográfica}

Não foi possível a observação direta do implante uma vez que a poliamida é um material radiolucente. Semelhante ao descrito por Carrillo et al. (2005), observou-se o espaço ocupado pela cinta e pelo seu sistema de fecho. Este ocupa um volume maior que o ocupado pelo sistema de fecho da cerclagem por fio de aço. 
Nos coelhos, observou-se osteólise discreta em 28,5\% dos animais do M60 tratados com náilon e em 14,3\% dos animais do M150 tratados com aço (Tabela 2). O fato pode eventualmente sugerir uma falha indesejável frente ao uso das cintas de náilon.
Em contrapartida, verificou-se que com a evolução do pós-operatório, a cerclagem tradicional de aço teve área de radiolucência levemente aumentada sugerindo que em uma aplicação em longo prazo, o implante de náilon apresenta recuperação mais rápida.

Tabela 2. Osteólise e aumento de volume e opacidade dos tecidos moles adjacentes: presença destes sinais clínicos indicando o número de animais e percentual (\%) em cada espécie animal, de acordo com o seu grupo e subgrupo experimental.

\begin{tabular}{cccccccccc}
\hline & \multicolumn{9}{c}{ Coelhos } \\
\cline { 2 - 10 } Variável & Grupo (M) & \multicolumn{2}{c}{ Náilon (SN) } & \multicolumn{2}{c}{ Aço (SA) } & \multicolumn{3}{c}{ Náilon (SN) } & \multicolumn{2}{c}{ Aço (SA) } \\
& & $\mathbf{0}$ & $\mathbf{1}$ & $\mathbf{0}$ & $\mathbf{1}$ & $\mathbf{0}$ & $\mathbf{1}$ & $\mathbf{0}$ & $\mathbf{1}$ \\
\hline \multirow{3}{*}{ Osteólise } & 30 & $7(100 \%)$ & $0(0 \%)$ & $7(100 \%)$ & $0(0 \%)$ & $5(100 \%)$ & $0(0 \%)$ & $5(100 \%)$ & $0(0 \%)$ \\
& 60 & $5(71,5 \%)$ & $2(28,5 \%)$ & $7(100 \%)$ & $0(0 \%)$ & $0(0 \%)$ & $4(100 \%)$ & $4(100 \%)$ & $0(0 \%)$ \\
& 150 & $7(100 \%)$ & $0(0 \%)$ & $6(85,7 \%)$ & $1(14,3 \%)$ & $3(60 \%)$ & $2(40 \%)$ & $5(100 \%)$ & $0(0 \%)$ \\
Volume e & 30 & $0(0 \%)$ & $7(100 \%)$ & $0(0 \%)$ & $7(100 \%)$ & $5(100 \%)$ & $0(0 \%)$ & $5(100 \%)$ & $0(0 \%)$ \\
Opacidade & 60 & $7(100 \%)$ & $0(0 \%)$ & $7(100 \%)$ & $0(0 \%)$ & $4(100 \%)$ & $0(0 \%)$ & $4(100 \%)$ & $0(0 \%)$ \\
& 150 & $5(71,5 \%)$ & $2(28,5 \%)$ & $7(100 \%)$ & $0(0 \%)$ & $5(100 \%)$ & $0(0 \%)$ & $5(100 \%)$ & $0(0 \%)$ \\
\hline
\end{tabular}

0 - ausência de osteólise, aumento de volume e opacidade; 1 - presença de osteólise, aumento de volume e opacidade.

Fonte: Elaboração dos autores.

Aumento de volume e opacidade dos tecidos moles adjacentes foi observado em 100\% dos membros pélvicos dos animais do M30 em ambos os subgrupos. De acordo com o descrito por Rihova (2000) e Sieminski e Gooch (2000) sobre a fase aguda da reparação óssea e integração dos implantes, a presença desta característica em 100\% dos membros pélvicos do M30 refere-se provavelmente a processo fisiológico de reparação, pois houve total resolução do mesmo nos momentos seguintes de avaliação.
Houve aumento de volume e opacidade dos membros pélvicos em dois coelhos no SN do M150, o que representa $28,5 \%$. Tal fato deve ter ocorrido por interferência externa, os quais eram muito agressivos e apresentaram feridas e abscesso por brigas, além de provável excesso de locomoção dos mesmo.

A reação periosteal está descrita na Tabela 3 . Houve apenas variação entre os subgrupos no M150, no qual foi observada reação levemente maior no SN em relação ao SA (Tabela 3). 
Tabela 3. Mediana e Valores mínimo e máximo da avaliação radiográfica segundo grupo e momento de avaliação em coelhos.

\begin{tabular}{cccccc}
\hline Reação periosteal & Grupo (M) & \multicolumn{5}{c}{ Subgrupos } \\
\cline { 2 - 5 } & & \multicolumn{2}{c}{ Náilon (SN) } & \multicolumn{2}{c}{ Aço (SA) } \\
\hline \multirow{3}{*}{ Cranial } & 30 & $1,0(1,0-2,0)$ & b B & $0,0(0,0-1,0)$ & a A \\
& 60 & $1,0(1,0-1,0)$ & b B & $0,0(0,0-1,0)$ & a A \\
& 150 & $0,0(0,0-0,0)$ & a A & $0,0(0,0-0,0)$ & a A \\
\hline \multirow{3}{*}{ Caudal } & 30 & $3,0(1,0-3,0)$ & b A & $2,0(1,0-3,0)$ & b A \\
& 60 & $2,0(2,0-3,0)$ & b A & $2,0(1,0-3,0)$ & ab A \\
& 150 & $0,0(0,0-3,0)$ & a A & $0,0(0,0-2,0)$ & a A \\
\hline \multirow{2}{*}{ Lateral } & 30 & $0,0(0,0-0,0)$ & a A & $0,0(0,0-0,0)$ & a A \\
& 60 & $1,0(0,0-3,0)$ & b A & $0,0(0,0-1,0)$ & a A \\
& 150 & $1,0(1,0-2,0)$ & b B & $0,0(0,0-0,0)$ & a A \\
\hline
\end{tabular}

Médias seguidas das mesmas letras minúsculas (coluna) e maiúsculas (linha) não diferem entre si pelo teste de Dunn (P $\leq 5 \%$ ) para os grupos e subgrupos, respectivamente. Escore: 0 - ausente; 1 - leve; 2 - moderado; 3 - intenso.

Fonte: Elaboração dos autores.

Nos ratos não foram observadas alterações quanto à osteólise nos membros do SA. Em todos os membros do SN do M60 e em 40\% do M150 observou-se a presença de osteólise (Tabela 2). Nos ratos, a osteólise observada apenas nos membros tratados com a cinta de náilon, no M60 e M150, sugere que tenha havido comprometimento circulatório na região da aplicação em decorrência da espessura da cinta de náilon em relação ao osso. Parham (1913) descreveu, após ter apresentado uma cinta de metal com superfície lisa, a existência de interferência no sistema circulatório cortical uma vez que esta interferia com o fluxo sanguíneo centrífugo, da medula para o periósteo. Para Albright, Johnson e Saha (1978) e Carrillo et al. (2005), a cerclagem tem o potencial de obstruir a saída do sangue venoso da córtex, mas a extensão dessa obstrução depende da área de contato entre o dispositivo da cerclagem e o osso.

Não foi observado aumento de volume e opacidade dos tecidos moles adjacentes em nenhum dos grupos ou subgrupos experimentais nos ratos (Tabela 2). Tanto no SN como no SA, não houve diferença na reação periosteal. Werner, Straughan e Vezin (1992) relataram que as cintas de náilon podem causar lesões e irritação. Uma irritação tecidual discreta pode ter ocorrido pela própria dimensão do implante (WERNER; STRAUGHAN; VEZIN, 1992); entretanto, o fio de náilon é considerado um material inerte não reativo aos tecidos; apesar disso, alguns indivíduos respondem a este material (PEARSON, 1970; SPACKMAN; CAYWOOD; JOHNSTON, 1984).

Nos ratos, na comparação entre subgrupos, na face lateral, verificou-se maior reação periosteal no SN do que no SA aos 30 e 150 dias de pósoperatório. SN apresentou também maior reação periosteal, na face medial, aos 60 dias (Tabela 4). Isto pode ter ocorrido pelo fato de a cinta de náilon, além de maior que o fio de aço, apresentar um sistema de trava saliente, após a sua colocação. Isso pode ter levado a uma agressão mecânica relativamente maior no local (WERNER; STRAUGHAN; VEZIN, 1992), a qual, não foi estatisticamente significativa nos períodos mais avançados. 
Tabela 4. Mediana e Valores mínimo e máximo da avaliação radiográfica segundo grupo e momento de avaliação em ratos.

\begin{tabular}{ccclcl}
\hline Reação periosteal & Grupo $(\mathbf{M})$ & \multicolumn{4}{c}{ Subgrupos } \\
\cline { 3 - 6 } & & \multicolumn{2}{c}{ Nylon (SN) } & Aço (SA) \\
\hline \multirow{3}{*}{ Lateral } & 30 & $1,0(1,0-2,0)$ & a B & $0,0(0,0-0,0)$ & a A \\
& 60 & $1,0(0,0-2,0)$ & a A & $0,0(0,0-0,0)$ & a A \\
& 150 & $1,0(1,0-1,0)$ & a B & $0,0(0,0-0,0)$ & a A \\
\hline \multirow{2}{*}{ Medial } & 30 & $1,0(0,0-2,0)$ & a A & $0,0(0,0-0,0)$ & a A \\
& 60 & $1,0(1,0-2,0)$ & a B & $0,0(0,0-0,0)$ & a A \\
& 150 & $1,0(0,0-2,0)$ & a A & $0,0(0,0-0,0)$ & a A \\
\hline
\end{tabular}

Médias seguidas das mesmas letras minúsculas (coluna) e maiúsculas (linha) não diferem entre si pelo teste de Dunn (P $\leq 5 \%$ ) para os grupos e subgrupos, respectivamente. Escore: 0 - ausente; 1 - leve; 2 - moderado; 3 - intenso.

Fonte: Elaboração dos autores.

\section{Avaliação macroscópica}

Nos coelhos houve maior proliferação de tecido fibroso ao redor do implante no $\mathrm{SN}$, nos momentos M30 e M60, quando comparados aos do M150 (Tabela 5). Quanto a esse parâmetro, foi notada uma tendência ao resultado, sem diferença significativa. Esta observação coincide com as afirmações feitas por Williams (1987), Sieminski e Gooch (2000), Rihova (2000), Ratner (2002) e Ratner e Bryant (2004) sobre a fase aguda dos mecanismos de reparação óssea e integração dos implantes, na qual ocorre um processo inflamatório com proliferação de tecido fibroso ao redor do implante. Já na fase crônica, ocorre proliferação de células ósseas (RATNER; BRYANT, 2004) e por consequência, como observado neste estudo, maior proliferação óssea ao redor do implante na fase final de observação (M150). No SA, verificou-se também esse mesmo comportamento quando comparado o grupo M30 com os M60 e M150. Não foram observadas diferenças entre o comportamento ósseo frente à aplicação das cintas de náilon e fio de aço.

Nos ratos não foram observadas alterações macroscópicas significativas quanto a fibrose e isso ocorreu provavelmente pela especificidade da espécie, bem como pelo menor tamanho, o que não acentua alterações mais discretas que possam ter ocorrido.

Tabela 5. Mediana e Valores mínimo e máximo da avaliação macroscópica segundo grupo e momento de avaliação em coelhos.

\begin{tabular}{cccccc}
\hline Macroscópica & Grupo (M) & \multicolumn{4}{c}{ Subgrupos } \\
\cline { 2 - 5 } & & \multicolumn{2}{c}{ Náilon (SN) } & Aço (SA) \\
\hline \multirow{3}{*}{ Tecido Fibroso } & 30 & $1,0(1,0-2,0)$ & b A & $1,0(1,0-2,0)$ & a A \\
& 60 & $2,0(1,0-3,0)$ & b A & $2,0(1,0-2,0)$ & a A \\
\multirow{2}{*}{ Aderência } & 150 & $0,0(0,0-3,0)$ & a A & $0,0(0,0-2,0)$ & a A \\
& 30 & $1,0(0,0-1,0)$ & a A & $0,0(0,0-1,0)$ & a A \\
& 60 & $1,0(1,0-2,0)$ & a A & $1,0(1,0-1,0)$ & a A \\
Neoformação óssea & 150 & $1,0(0,0-3,0)$ & a A & $1,0(0,0-2,0)$ & a A \\
& 30 & $1,0(1,0-1,0)$ & a A & $1,0(1,0-2,0)$ & a A \\
& 60 & $1,0(1,0-2,0)$ & a A & $2,0(1,0-3,0)$ & b A \\
\hline
\end{tabular}

Médias seguidas das mesmas letras minúsculas (coluna) e maiúsculas (linha) não diferem entre si pelo teste de Dunn (P $\leq 5 \%$ ) para os grupos e subgrupos, respectivamente. Escore: 0 - ausente; 1 - leve; 2 - moderado; 3 - intenso.

Fonte: Elaboração dos autores. 


\section{Biometria dos fêmures}

Entre os grupos e subgrupos não houve diferença estatística nas medidas morfométricas: distância do trocânter menor à epífise distal e distância da face cranial à caudal. Na distância da face medial à lateral não houve diferença estatística entre os subgrupos, porém verificou-se aumento desta dos
30 para os 150 dias em ambos os subgrupos (Tabelas 6 e 7). Estes resultados sugerem que não tenha ocorrido interferência na morfometria dos fêmures, uma vez que nenhuma das medidas do M150 se mostrou inferior às do M30. Não foram encontradas na literatura consultada dados de morfometria relacionados ao uso de cerclagens.

Tabela 6. Média e desvio-padrão das medidas do fêmur segundo grupo e momento de avaliação em coelhos.

\begin{tabular}{cccccc}
\hline Medidas do Fêmur & Grupo (M) & \multicolumn{5}{c}{ Subgrupos } \\
\cline { 2 - 6 } & & \multicolumn{2}{c}{ Náilon (SN) } & Aço (SA) \\
\hline \multirow{2}{*}{ Distância Trocânter } & 30 & $73,30 \pm 2,09$ & a A & $74,06 \pm 1,33$ & a A \\
Menor-Epífise distal & 60 & $73,42 \pm 2,90$ & a A & $73,19 \pm 2,90$ & a A \\
& 150 & $74,66 \pm 1,52$ & a A & $74,94 \pm 2,27$ & a A \\
\hline \multirow{3}{*}{ Distância Crânio-caudal } & 30 & $7,97 \pm 0,49$ & a A & $8,01 \pm 0,80$ & a A \\
& 60 & $8,59 \pm 0,57$ & a A & $8,56 \pm 0,78$ & a A \\
& 150 & $8,07 \pm 0,44$ & a A & $8,21 \pm 0,45$ & a A \\
Distância Medio-lateral & 30 & $9,51 \pm 0,68$ & a A & $9,36 \pm 0,38$ & a A \\
& 60 & $10,20 \pm 0,60$ & ab A & $10,05 \pm 0,92$ & ab A \\
& 150 & $10,91 \pm 0,69$ & b A & $10,49 \pm 0,54$ & b A \\
\hline
\end{tabular}

Médias seguidas das mesmas letras minúsculas (coluna) e maiúsculas (linha) não diferem entre si pelo teste de Dunn (P $\leq 5 \%$ ) para os grupos e subgrupos, respectivamente. Escore: 0 - ausente; 1 - leve; 2 - moderado; 3 -intenso.

Fonte: Elaboração dos autores.

Tabela 7. Média e desvio-padrão das medidas do fêmur segundo grupo e momento de avaliação em ratos.

\begin{tabular}{cccccc}
\hline Medidas do Fêmur & Grupo (M) & \multicolumn{4}{c}{ Subgrupos } \\
\cline { 3 - 6 } & & \multicolumn{2}{c}{ Náilon (SN) } & Aço (SA) \\
\hline \multirow{2}{*}{ Distância Trocânter } & 30 & $24,90 \pm 0,80$ & a A & $24,62 \pm 0,96$ & a A \\
Menor-Epífise distal & 60 & $26,74 \pm 0,61$ & b A & $26,90 \pm 0,48$ & b A \\
& 150 & $26,80 \pm 0,60$ & b A & $26,70 \pm 1,18$ & b A \\
\hline \multirow{2}{*}{ Diâmetro Crânio-caudal } & 30 & $4,22 \pm 0,74$ & ab A & $4,80 \pm 0,19$ & b A \\
& 60 & $4,90 \pm 0,12$ & b A & $4,65 \pm 0,47$ & b A \\
& 150 & $3,76 \pm 0,27$ & a A & $3,78 \pm 0,11$ & a A \\
\hline \multirow{2}{*}{ Diâmetro Latero-lateral } & 30 & $3,80 \pm 0,25$ & b A & $3,6 \pm 0,10$ & b A \\
& 60 & $3,80 \pm 0,23$ & b A & $3,90 \pm 0,46$ & b A \\
& 150 & $3,34 \pm 0,20$ & a A & $3,18 \pm 0,15$ & a A \\
\hline
\end{tabular}

Médias seguidas das mesmas letras minúsculas (coluna) e maiúsculas (linha) não diferem entre si pelo teste de Dunn (P $\leq 5 \%$ ) para os grupos e subgrupos, respectivamente. Escore: 0 - ausente; 1 - leve; 2 - moderado; 3 -intenso.

Fonte: Elaboração dos autores.

Após aplicação dos implantes nos fêmures dos ratos, a única medida que não foi afetada foi a distância do trocânter menor à epífise distal sugerindo um crescimento normal em comprimento. Já o diâmetro do osso sofreu uma diminuição acentuada do M30 para o M150, o que pode eventualmente sugerir que tenha havido comprometimento na microvasculatura periosteal 
ou mesmo por impedimento mecânico, uma vez que os ossos dos ratos são menores e mais frágeis.

\section{Avaliação histopatológica}

Carrillo et al. (2005), em seu estudo sobre a utilização da cinta de náilon comercial associada a pinos intramedulares na osteossíntese de fêmures de coelhos, observaram que o dispositivo submetido à autoclavagem foi biocompatível com a espécie, não sendo verificados quadros histológicos compatíveis com processo inflamatório ou tóxico. Nos coelhos do SA verificou-se, no M30, maior quantidade de fibrose do que nos M60 e M150.
Entretanto, não houve diferença entre os dois subgrupos para todas as variáveis histológicas avaliadas (fibrose, porosidade, proliferação, isquemia, atividade osteoblástica e maturidade óssea sob o implante) nos diferentes momentos de avaliação. No M30, a atividade osteoblástica foi mais intensa em relação aos demais grupos (M60 e M150) no SN, sendo também evidenciada maturidade óssea progressiva do M30 para o M150 (Tabela 8). No coelho ${ }^{\circ} 6$ do $\mathrm{SN}$ foi possível a observação do material plástico piogranulomatoso (macrófagos, neutrófilos e linfócitos) na área do implante. Achados estes compatíveis com o processo traumático já descrito.

Tabela 8. Mediana e Valores mínimo e máximo da avaliação histopatológica segundo grupo e momento de avaliação em coelhos.

\begin{tabular}{|c|c|c|c|c|c|}
\hline \multirow[t]{2}{*}{ Histopatológica } & \multirow[t]{2}{*}{ Grupo (M) } & \multicolumn{4}{|c|}{ Subgrupos } \\
\hline & & \multicolumn{2}{|c|}{ Náilon (SN) } & \multicolumn{2}{|c|}{ Aço (SA) } \\
\hline \multirow{3}{*}{ Fibrose } & 30 & $2,0(1,0-3,0)$ & a A & $2,0(0,0-3,0)$ & $\mathrm{bA}$ \\
\hline & 60 & $1,0(0,0-3,0)$ & a A & $0,0(0,0-1,0)$ & a A \\
\hline & 150 & $1,0(0,0-3,0)$ & a A & $0,0(0,0-1,0)$ & a A \\
\hline \multirow{3}{*}{ Porosidade } & 30 & $1,0(0,0-2,0)$ & a A & $0,0(0,0-1,0)$ & a A \\
\hline & 60 & $1,0(0,0-2,0)$ & a A & $0,0(0,0-1,0)$ & a A \\
\hline & 150 & $1,0(0,0-1,0)$ & a A & $0,0(0,0-0,0)$ & a A \\
\hline \multirow{3}{*}{$\begin{array}{c}\text { Atividade } \\
\text { Osteoblástica }\end{array}$} & 30 & $2,0(1,0-3,0)$ & $\mathrm{bA}$ & $2,0(0,0-2,0)$ & a A \\
\hline & 60 & $1,0(0,0-2,0)$ & a A & $0,0(0,0-1,0)$ & a A \\
\hline & 150 & $1,0(0,0-2,0)$ & a A & $1,0(0,0-2,0)$ & a A \\
\hline \multirow{3}{*}{$\begin{array}{l}\text { Maturidade sob. o } \\
\text { implante }\end{array}$} & 30 & $2,0(2,0-2,0)$ & $\mathrm{aA}$ & $2,0(2,0-3,0)$ & a A \\
\hline & 60 & $2,0(2,0-3,0)$ & $\mathrm{ab} A$ & $3,0(2,0-3,0)$ & a A \\
\hline & 150 & $3,0(1,0-3,0)$ & $\mathrm{b} A$ & $3,0(1,0-3,0)$ & a A \\
\hline
\end{tabular}

Médias seguidas das mesmas letras minúsculas (coluna) e maiúsculas (linha) não diferem entre si pelo teste de Dunn (P $\leq 5 \%$ ) para os grupos e subgrupos, respectivamente. Escore: 0 - ausente; 1 - leve; 2 - moderado; 3 - intenso.

Fonte: Elaboração dos autores.

Nos ratos observou-se variação, no exame histopatológico, apenas na presença de fibrose no momento 150, que foi mais acentuada no SA em relação ao SN (Tabela 9). Apesar da radiolucência verificada nos membros tratados com náilon, ao exame histopatológico foi detectada presença discreta de isquemia tanto no SN quanto no SA, sem diferença, portanto, entre os dois membros. 
Tabela 9. Mediana e Valores mínimo e máximo da avaliação histopatológica segundo grupo e momento de avaliação em ratos.

\begin{tabular}{|c|c|c|c|c|c|}
\hline \multirow[t]{2}{*}{ Histopatológica } & \multirow{3}{*}{$\begin{array}{c}\text { Grupo (M) } \\
30\end{array}$} & \multicolumn{4}{|c|}{ Subgrupos } \\
\hline & & \multicolumn{2}{|c|}{ Náilon (SN) } & \multicolumn{2}{|c|}{ Aço (SA) } \\
\hline \multirow{3}{*}{ Fibrose } & & $1,0(0,0-2,0)$ & a A & $0,0(0,0-1,0)$ & a A \\
\hline & 60 & $1,0(0,0-3,0)$ & a A & $1,5(0,0-3,0)$ & a A \\
\hline & 150 & $1,0(0,0-3,0)$ & a A & $2,0(2,0-3,0)$ & $\mathrm{b} B$ \\
\hline \multirow{3}{*}{ Porosidade } & 30 & $0,0(0,0-0,0)$ & a A & $0,0(0,0-1,0)$ & a A \\
\hline & 60 & $0,0(0,0-0,0)$ & a A & $0,0(0,0-0,0)$ & a A \\
\hline & 150 & $1,0(0,0-2,0)$ & $\mathrm{aA}$ & $1,0(0,0-2,0)$ & a A \\
\hline \multirow{3}{*}{ Proliferação } & 30 & $1,0(0,0-2,0)$ & a A & $1,0(0,0-2,0)$ & a A \\
\hline & 60 & $0,5(0,0-2,0)$ & a A & $1,0(1,0-1,0)$ & a A \\
\hline & 150 & $0,0(0,0-1,0)$ & $\mathrm{aA}$ & $0,0(0,0-1,0)$ & a A \\
\hline \multirow{3}{*}{ Isquemia } & 30 & $0,0(0,0-2,0)$ & a A & $0,0(0,0-1,0)$ & a A \\
\hline & 60 & $0,0(0,0-0,0)$ & a A & $0,0(0,0-0,0)$ & a A \\
\hline & 150 & $0,0(0,0-0,0)$ & a A & $0,0(0,0-0,0)$ & a A \\
\hline \multirow{3}{*}{ Ativ. Osteoblástica } & 30 & $0,0(0,0-2,0)$ & a A & $0,0(0,0-1,0)$ & a A \\
\hline & 60 & $1,0(1,0-2,0)$ & a A & $1,0(0,0-2,0)$ & a A \\
\hline & 150 & $1,0(0,0-2,0)$ & $\mathrm{aA}$ & $1,0(1,0-2,0)$ & a A \\
\hline \multirow{3}{*}{$\begin{array}{l}\text { Maturidade sob. o } \\
\text { implante }\end{array}$} & 30 & $3,0(2,0-3,0)$ & a A & $2,0(2,0-3,0)$ & a A \\
\hline & 60 & $2,0(1,0-3,0)$ & a A & $2,0(1,0-3,0)$ & a A \\
\hline & 150 & $3,0(2,0-3,0)$ & $\mathrm{aA}$ & $2,0(1,0-2,0)$ & a A \\
\hline
\end{tabular}

Médias seguidas das mesmas letras minúsculas (coluna) e maiúsculas (linha) não diferem entre si pelo teste de Dunn (P $\leq 5 \%$ ) para os grupos e subgrupos, respectivamente. Escore: 0 - ausente; 1 - leve; 2 - moderado; 3 - intenso.

Fonte: Elaboração dos autores.

As alterações circulatórias podem, mais uma vez, ser suportadas pela observação e relato feito por Carrillo et al. (2005), os quais afirmam que a cerclagem tem o potencial de obstruir a saída do sangue venoso da córtex, mas a extensão dessa obstrução depende da área de contato entre o dispositivo da cerclagem e o osso. Considerando que se for feita uma comparação percentual entre a largura da cinta e o comprimento dos fêmures, desde o trocânter menor à epífise distal, nos coelhos, ela representa 3,4\% do comprimento e nos ratos, 9,4\%. Assim, a probabilidade de ocorrência de lesão por lesão vascular foi maior ratos nos que nos coelhos. Sugere-se que a largura da cinta de náilon deva ser um fator importante no momento do procedimento cirúrgico e que esta, de preferência, não chegue a valores próximos a $9 \%$ da distância do trocânter menor à epífise distal.

Conclui-se que, as abraçadeiras de náilon quando utilizadas em torno da diáfise femoral de coelhos e ratos mimetizando a aplicação das cerclagens de aço, são biocompatíveis e não desencadeiam reação de corpo estranho. Novos estudos precisam ser realizados em pacientes com fraturas em que a cerclagem se encaixe como tratamento adjuvante na osteossíntese, bem como testes de tração e elasticidade da cinta.

\section{Aspectos Éticos e de Biossegurança}

Todos os procedimentos experimentais foram aprovados pela Câmara de Ética em Experimentação Animal da FMVZ - Unesp - Botucatu, sob o protocolo n ${ }^{\circ}$ 94/2007. 


\section{Referências}

ALBRIGHT, J. A.; JOHNSON, T. R.; SAHA, S. Effects on blood supply. In: GHISTA, D. N.; ROAF, R. Orthopaedic mechanics: procedures and devices. New York and San Francisco: Academic Press, 1978. p. 181182.

AlBUQUeRQUE, J. A. C. O plástico na prática. Porto Alegre: Sagra, 1990. 153 p.

CARRILLO, J. M.; SOPENA, J. J.; RUBIO, M.; REDONDO, J. I.; SERRA, I.; SOLER, C. Experimental use of polyamide bands in combination with intramedullary pinning for repair of oblique femoral fractures in rabbits. Veterinary Surgery, Davis, v. 34, n. 4, p. 387-392, 2005.

FRANÇA, R. O. Ovariectomia e orquiectomia em eqüinos: uso da abraçadeira de náilon na hemostasia preventiva em comparação ao categute e emasculador. 2005. Dissertação de Mestrado em Ciência Animal. Escola de Veterinária da Universidade Federal de Goiás, Goiânia. Disponível em: <http://ppgca.vet.ufg.br/ uploads/67/original_Dissertacao2005_Rodrigo_Franca. pdf $>$. Acesso em: 11 dez. 2012.

KIRBY, B. M.; WILSON, J. W. Effect of circunferential bands on cortical vascularity and viability. Journal of Orthopedic Research, New Orleans, v. 9, n. 2, p. 174179, 1991.

LIMA, A. F. F.; LUNA, S. P. L.; RODRIGUES, M. M. P.; QUITZAN, J. G. Avaliação histológica e videolaparoscópica de ligaduras dos pedículos ovarianos realizados com mononáilon agulhado ou abraçadeiras auto-estáticas de náilon em cadelas submetidas à ovariossalpingohisterectomia pela técnica do gancho. Ars Veterinaria, Jaboticabal, v. 26, n. 2, p. 66-70, 2010.

MIRANDA, A. H.; SILVA, L. A. F.; TAVARES, G. A.; AMARAL, A. V. C.; MIRANDA, H. G. Abraçadeira de náilon: Resistência à tração em testes físicos e seu emprego como cerclagem no fêmur de cães. Ciência Animal Brasileira, Goiânia, v. 7, n. 3, p. 299-307, 2006.

MIRANDA, A. H.; SILVA, L. A. F.; TAVARES, G. A.; LIMA, A. M. V.; AMARAL, A. V. C.; MIRANDA, H. G.; FRANCO, L. G.; ROCHA, L. A.; OLIVEIRA, K. S.; SILVA, E. B. Avaliação da resistência da abraçadeira de náilon utilizada como cerclagem na redução de fraturas de cães. Ciência Animal Brasileira. Goiânia, v. 5, p. 199201, 2004. Suplemento.

MUZZI, L. A. P.; REZENDE, C. M. F.; MUZZI, R. A. L.; BORGES, N. F. Ruptura do ligamento cruzado cranial em cães: fisipatologia e diagnóstico. Clínica Veterinária, São Paulo, v. 46, n. 1, p. 32-42, 2003.
PARHAM, F. W. A new device for treatment of fractures. New Orleans Med. Surgery Journal, New Orleans, v. 66, p. $465-467,1913$.

PEARSON, H. Ovariohysterectomy in the bitch. The Veterinary Record, London, v. 87, n. 21, p. 646-647, 1970.

RAISER, A. G.; ROJAS, R. G.; PIPPI, N. L.; CARDOSO, G. Influência dos implantes metálicos e traumatismos na etiologia de tumores ósseos. A Hora Veterinária, Porto Alegre, v. 3, p. 17-19, 1981.

RATNER, B. D. Reducing capsular thickness and enhancing angiogenesis around implant drug release systems. Journal of Controlled Release, Boston, v. 8, n. 1-3, p. 211-218, 2002.

RATNER, B. D.; BRYANT, S. J. Biomaterials: where we have been and where we are going. Annual Review of biomedical Engineering, Palo Alto, v. 6, n. 1, p. 41-75, 2004.

RHINELANDER, F. W.; STEWART, C. L. Experimental fixation of femoral osteotomies by cerclage with nylon straps. Clinical Orthopaedics and Related Research, Philadelphia, v. 179, p. 298-307, 1983.

RIHOVA, B. Immunocompatibility and biocompatibility of cell delivery systems. Advanced Drug Delivery Reviews, Salt Lake City, v. 42, n. 1-2, p. 65-80, 2000.

SCHMIDT, T. L.; DAVIS, W. M. Intraoperative use of nylon bands in fracture fixation. Clinical Orthopaedics and Related Research, Philadelphia, v. 154, n. 1, p. 341343, 1981.

SIEMINSKI, A. L.; GOOCH, K. J. Biomaterialmicrovasculature interactions. Biomaterials, Philadelphia, v. 21, n. 22, p. 2233-2241, 2000.

SILVA, L. A. F.; ARAÚJO, G. R. S.; MIRANDA, A. H.; RABELO, R. E.; GARCIA, A. M.; SILVA, O. C.; ARAÚJO, I. F.; MACEDO, S. P.; SOUSA, J. N.; FIORAVANTI, M. C.; OLIVEIRA, K. S.; AMARAL, A. V. C.; SILVA, E. B. Ovariohisterectomia em cadelas: uso da abraçadeira de náilon da hemostasia preventiva. Ciência Animal Brasileira, Goiânia, v. 5, p. 100-102, 2004, Suplemento.

SORBELLO, A. A.; GIUDUGLI, J. N.; ANDRETTO, R. Nova alternativa para ligaduras em cirurgias videoendoscópicas ou convencionais, com emprego de fitas de nylon em estudo experimental. Revista Brasileira de Coloproctologia, Rio de Janeiro, v. 19, n. 1, p. 24-26, 1999.

SPACKMAN, C. J. A.; CAYWOOD, D. D.; JOHNSTON, G. R. Granulomas of the uterine and ovarian stumps: a case report. American Animal Hospital Association, New York, v. 20, n. 3, p. 449-453, 1984. 
WERNER, R. E.; STRAUGHAN, A. J.; VEZIN, D. Nylon cable band reactions in ovariohysterectomized biches. Journal of the American Veterinary Medical Association, New York, v. 200, n. 1, p. 64-66, 1992.
WILLIAMS, D. F. Definitions in biomaterials. In: Progress in biomedical engineering. New York: Elsevier, 1987. p. 49-59.

ZAR, J. H. Biostatistical analysis. 4. ed. Upper Saddle River, NJ: Prentice Hall, 1999. 662 p. 